\title{
EVALUATING A $p$-TH ORDER COHOMOLOGY OPERATION
}

\author{
J. HARPER AND A. ZABRODSKY
}

\begin{abstract}
A certain $p$-th order cup product is detected by a $p-t h$ order cohomology operation. The result is applied to fnite $H$-spaces, to show that several properties of compact lie groups do not hold for arbitrary torsion free finite $H$-spaces.
\end{abstract}

\section{Summary of results}

In this paper we give the detailed computations of results announced in [3]. As the computations involve many technical details we try to present the main line of proof separately in Chapter 2-filling in the missing details in Chapters 3-7. In Chapter 8 we bring some applications.

Let $p$ be a prime. Throughout this paper we write $H^{*}(X)=H^{*}(X, \mathbf{Z} / p \mathbf{Z})$. Let $\phi_{n}$ denote an $n+1$ order $\bmod p$ cohomology operation associated with the Toda brackets $\langle\overbrace{p^{a}, \ldots, p^{p-1}, p^{2}}^{n+1}\rangle(a=1$ if $n$ is even $a=p-1$ if $n$ is odd $)$. By this we mean a stable cohomology operation universally defined on a spectra

Partially supported by grants of the U.S.-Israel Binational Science Foundation and the U.S. National Science Foundation.

The editor regrets very much to inform that Professor Zabrodsky died on November 86 .

The frrst author dedicates this paper to his memory. 
$E_{n}$ with a Postrikov system given by:

(D1)

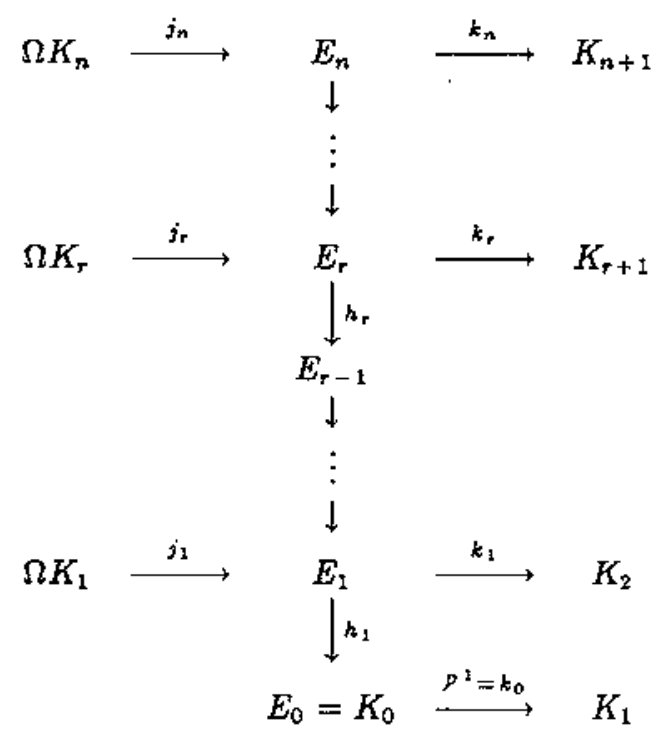

with the properties:

1.0.

(1) $K_{0}=K(\mathbf{Z} / p \mathbf{Z}, 0)$-the Eilenberg MacLane Spectrum.

(2) for $r \geq 1, \quad K_{r}=\sum^{i} K_{0}, \quad t_{r}= \begin{cases}s p q+q-2 s & r=2 s+1 \\ s p q-2 s+1 & 0<r=2 s\end{cases}$ $q=2 p-2$.

(3) $\Omega K_{r} \stackrel{i_{r}}{\longrightarrow} E_{r} \stackrel{h_{r}}{\longrightarrow} E_{r-1}$ and for $0 \leq r<n E_{r+1} \stackrel{h_{r+1}}{\longrightarrow} E_{r} \stackrel{K_{r}}{\longrightarrow} K_{r+1}$ are (co) fibrations.

(4) $\left[k_{r} \circ j_{r}\right] \in\left[\Omega K_{r}, K_{r+1}\right]=p a_{r} \in \underline{A}(p)$ where

$$
a_{r}= \begin{cases}1 & \text { for } r \text { - even } \\ p-1 & \text { for } r \text { - odd }\end{cases}
$$

$\phi_{n}$ is then given by the universal example (in the sense of $\left.[\mathbf{1}]\right)\left\langle x=H^{0}\left(h_{1} \circ \ldots\right.\right.$ $\left.\left.\left.\circ h_{n}\right) v, E_{n}, u=H^{t_{n+1}}\left(k_{n+1}\right)\left(\Sigma^{t_{n+1}}\right)\right)\right\rangle$.

1.1. Rernark. We shall use the notations of D1 to describe a non stable (say $m-1$ connected) representative of $\phi_{n}$. In that case the maps in (3) are fibrations, $K_{0}=K(\mathbf{Z} / p \mathbf{Z}, m), K_{r}=K\left(\mathbf{Z} / p \mathbf{Z}, m+t_{r}\right)$.

The main purpose of this paper is to show:

The Main Theorem.

(1) $\phi_{p-1}$ exists and if $n \not \equiv(-1) \bmod p$ then $p^{n} \imath_{2 n+1} \in H^{2 n p+1}(K(\mathbf{Z} / p Z$, $2 n+1)$ ) is in the domain of $\phi_{p-1}$ and $\imath_{2 n+1} \cdot P^{1} \imath_{2 n+1} \cdot p^{2} \imath_{2 n+1} \cdot \ldots \cdot p^{p-1_{2 n+1}} \in$ $\in \phi_{p-1}\left(p^{n} i_{2 n+1}\right)$. 
(2) $0 \notin \phi_{p-1}\left(p^{n} t_{2 n+1}\right)$.

The principal applications of the main theorem (which generalizes results in [11]) is the construction of counterexamples that the following properties shared by Lie groups, are not valid in general for finite $\mathrm{H}$-spaces:

\subsection{Examples.}

1.2.1. Let $X$ be a compact connected Lie group. If $H^{*}(X) \approx \wedge\left(x_{2 m_{1}+1}, \ldots\right.$ $\left.\ldots, x_{2 m_{r}+1}\right), m_{1} \leq m_{2} \leq \cdots \leq m_{r}$ then there exists a map $f: X \rightarrow S^{2 m_{r+1}}$ with $x_{2 m+1} \in \operatorname{Im} H^{*}(f)$.

1.2.2. It was recently proved by $\mathrm{H}$. Miller ([4]) that for $X=O(n), U(n), S p(n)$ (and consequently $X$ may belong to a larger family of Lie groups) $X$ splits stably according to the (integral) algebraic weight of its generators, i.e. if $H^{*}(X)$ is as above $\Sigma^{\infty} X \approx Y_{1} \vee Y_{2} \vee \cdots \vee Y_{r}$ where $H^{*}\left(Y_{j}\right)$ contains all the monomials $x_{2 m_{t_{2}}+1} \cdot x_{2 m_{t_{1}+1}} \cdot \ldots \cdot x_{2 m_{t_{j}}+1}$ in $H^{*}(X)$.

1.2.3. It is also well known (e.g: see $[2$, theorem 1.1] with $f=\Sigma T, T$ - the $\lambda$-th power map) that for any H-space $X$ with $H^{*}(X)$ as above $\Sigma X \approx Y_{1} \vee$ $\vee Y_{2} \vee \cdots \vee Y_{p-1}$ where $H^{*}\left(Y_{j}\right)$ contains all the monomials in the $x_{2 m,+}$ 's of length $j \bmod p-1$ (these will be called elements of $\bmod p-1$ algebraic weight j).

Thus, the following question naturally arises:

1.3. (See e.g: [9, problem 14]) Which of these Lie groups properties are shared by finite $\mathrm{H}$-spaces in general?

1.3.1. If $X$ is a mod $p H$-space with

$$
H^{*}(X)=\wedge\left(x_{2 m_{1}+1}, x_{2 m_{3}+1}, \ldots, x_{2 m_{+}+1}\right)
$$

does there exist a map $f: X \longrightarrow S^{2 m_{r+1}}$ with $x_{2 m+2} \in \operatorname{Im} H^{*}(f)$ ?

1.3.2. Is there a mod $p$ stable splitting of a mod $p$ finite $H$-space according to the (integral) algebraic weight of its cohomology?

1.3.3. (The weakest common property): Let $X$ be as in 1.3.2. Is there a stable $\operatorname{map} \hat{f}: X \longrightarrow \Omega^{\infty} \Sigma^{\infty} S^{2 m+1} \quad$ with $x_{2 m+1} \in \operatorname{In} H^{*}(\hat{f})$ ?.

All these questions are negatively answered by the following:

1.4. Example. (See [3]): There exists a $\bmod p H$-space $(p \geq 5) X$ with $H^{*}(X)=\wedge\left(x_{2 p+1}, p^{1} x_{2 p+1}, \ldots, p^{p} x_{2 p+1}\right)$. It follows from the main theorem that there is no stable map $f: X \longrightarrow \Omega^{\infty} \Sigma^{\infty} S^{p^{2}+1}$ with $p^{p} x_{2 p+1} \in \operatorname{Im} H^{*}(f)$. (See Corollary 8.6)

A consequence of the above example (and similar $\infty$ dimensional examples) is that for an arbitrary stable splitting of $K(Z / p Z, 2 n+1)(n \not \neq-1(p))$ any summand containing $P^{n} i_{2 n+1}$ in its cohomology will have to include the product $\imath_{2 n+1} \cdot p^{1} \imath_{2 n+1} \cdot \ldots \cdot p^{p-1} i_{2 n+1}$.

Finally one can deduce a non realizability theorem: 
1.5. Proposition. (See Corollary 8.5) If $n \not \equiv-1$ mod $p$ then there is no $\bmod p H$-space (or even a $\lambda$-power space) $X$ with

$$
H^{*}(X)=\wedge\left(x_{2 n+1}, P^{1} x_{2 n+1}, \ldots, p^{p-1} x_{2 n+1}\right)
$$

If $n$ is large enough there is no space with the above cohomology. (Note that $\wedge\left(x_{2 p-1}, p^{1} x_{2 p-1}, \ldots, p^{p-1} x_{2 p-1}\right)$ is the mod $p$ cohomology of one of Nishida's factors of $S U\left(p^{2}-p+1\right)([7 /)$. Hence $n \not \equiv-1(p)$ is essential).

\section{Outline of proof}

Evaluating a $p$-th order cohomology operation cannot be expected to be an easy task. Generally it involves many technical details. Still, the core of the computations has a definite straight forward line of thought. In this section we shall try to outline the proof of our main results referring the reader to the auxiliary technical computations in the following sections. The first observation (3.1) is that $\phi_{p-1}$ exists. (This is obvious for $p=2$ thus we restrict ourselves to $p$-odd). The reason is quite simple: Assuming $\phi_{r-1}$ exists, the obstruction for the existence of $\phi_{r}$ is a class in the cohomology of $E_{r-1}$. It turns out that for small $r$ the cohomology of $E_{r-1}$ in the appropriate dimension is zero.

The fact that $p^{n} \imath_{2 n+1}$ is in the domain of $\phi_{p-1}$ is embedded in the inductive proof of the main theorem but essentially it is a consequence of 4.3 .

The principal idea of the proof of the main theorem is as follows: Let $K=$ $=K(\mathbf{Z} / p \mathbf{Z}, 2 n+1)$ and let

$$
\Sigma K=B_{2} \subset B_{2} \subset B_{3} \subset \cdots \subset B_{\infty}=B K=K(\mathbf{Z} / p \mathbf{Z}, 2 n+2)
$$

be the Minor-Rothenberg-Steenrod-Milgram filtration $([5],[8])$ of the classifying space of $K$. We shall use the following notations:

2.0.

(i) $i_{r_{s} s}: B_{r} \longrightarrow B$, is the inclusion, with the abreviation $\dot{z}_{r_{1}+1}=i_{r}$.

(ii) $B_{\mathrm{r}} \cup C B_{r-1}=B_{r}, B_{r-1}, j_{r}: B_{r} \longrightarrow B_{r}, B_{r-1}$ denotes the inclusion.

(iii) $\delta_{r}: B_{r}, B_{r-1} \longrightarrow \Sigma_{r-1} B_{r-1}$ the third leg of the Puppe sequence.

(iv) $z=\imath_{2 n+2}$ denotes all fundamental classes in $H^{2 n+2}\left(B_{r}\right), \quad 1 \leq r \leq \infty$.

We evaluate $\phi_{p-1}$ on $p^{n} \tau_{2 n+2} \in H^{2 n p+2}\left(\sum K\right)=H^{2 n p+2}\left(B_{1}\right)$ using a $2 n p+1$ connected non stable version of (D1). Thus one reads D1 with $K_{r}=K(\mathbf{Z} / p \mathbf{Z}$, $\left.2 n p+2+t_{r}\right)\left(t_{0}=0\right), t_{r}$ as defined in $0.1-(2)$.

We start an inductive process: 


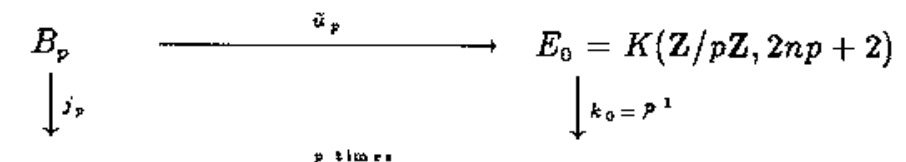

$(\mathrm{D} 2)_{p}$

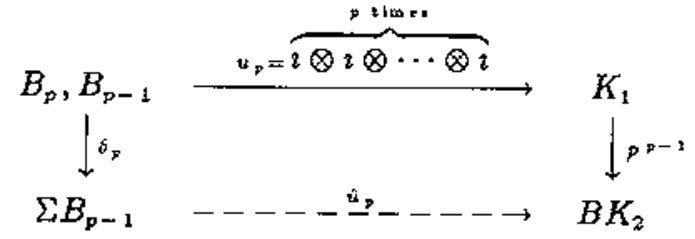

$$
n \not \equiv-1(\bmod p)
$$

$\left[\bar{u}_{p}\right]=\frac{1}{n+1} p^{n} i_{2 n+2}$, so $P^{1}\left[\tilde{u}_{p}\right] z_{2 n+2}=p^{n+1} i_{2 n+2}=i_{2 n+2}^{p}=\left[j_{p}\right](\imath \otimes \imath \otimes \cdots \otimes \imath)=$ $r$ times

$=\left[u_{p} \circ j_{p}\right]$. Note that $H^{*}\left(B_{r}, B_{r-1}\right) \approx \overbrace{\Sigma H^{*}(K) \otimes \Sigma H^{*}(K) \otimes \cdots \otimes \Sigma H^{*}(K)}$ thus the notation $u_{p}=\imath \otimes \cdots \otimes$. Also note that the map induced by $\sum_{i_{r-1}} \circ \delta_{r}$ from $H^{\bullet}\left(B_{r-1}, B_{r-2}\right)$ to $H^{\bullet}\left(B_{r}, B_{r-1}\right)$ corresponds to $d_{r-1}: \underline{B}_{r-1} \longrightarrow \underline{B}$, in the cobar construction of $A=H^{*}(K)$ (with the appropriate shift of dimension).

Any choice of homotopies $W: k_{0} \circ \tilde{u}_{p} \sim u_{p} \circ j_{p}, \ell: * \sim p^{p-1} \circ k_{0}$ induces a map $\hat{u}_{p}: \Sigma B_{p-1} \longrightarrow B K_{2}$ and its adjoint $\hat{u}_{p \#}: B_{p-1} \longrightarrow K_{2}$ factors up to homotopy as $B_{p-1} \stackrel{\dot{u}_{p-1}}{\longrightarrow} E_{1} \stackrel{k_{1}}{\longrightarrow} K_{2}$, hence $\hat{u}_{p \#}$ represents $\phi_{1}\left(p^{n} \imath_{2 n+2}\right)$. To continue the inductive step one has to show that $\hat{u}_{p \#}$ factors (up to homotopy) as

$$
B_{p-1} \stackrel{j_{p-1}}{\longrightarrow} B_{p-1}, B_{p-2} \stackrel{u_{p-2}}{\longrightarrow} K_{2}
$$

Inductively, suppose one has a (homotopy) commutative diagram (for $3 \leq r \leq$ $\leq p)$

(D2).

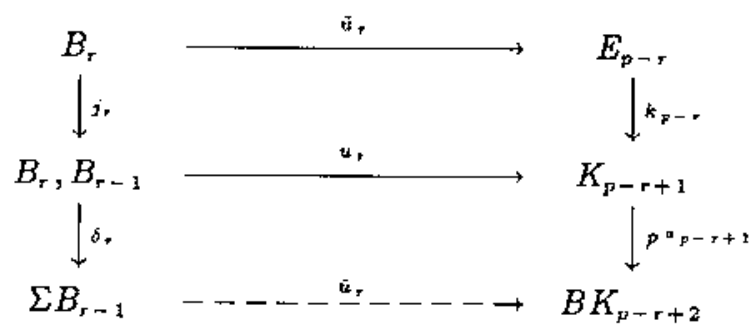

where $\left[k_{p-r} \circ \bar{u}_{r}\right]$ represents $\phi_{p-r}\left(P^{n} z_{2 n+2}\right)$. Any choice of homotopies $W: k_{p-r} \circ$ $\tilde{u}_{r} \sim u_{r} \circ j_{r}$ and $\ell: * \sim p^{a_{p-r+1}} \circ k_{p-r}$ (which exists by 3.1) induces a map $\hat{u}_{r}: \Sigma B_{r-1} \longrightarrow B K_{p-r+2}$ whose adjoint $\left(\hat{u}_{r}\right)_{\#}$ factors as $B_{r-1} \stackrel{\mathfrak{u}_{r-1}}{\longrightarrow} E_{p-r+1}$ $\stackrel{k_{p-r+2}}{\longrightarrow} K_{p-r+2}$, hence, $\left(\hat{u}_{r}\right)_{\#}$ represents $\phi_{p-r+1}\left(p^{n} t_{2_{n+2}}\right)$. 
To proceed inductively one has to show that $\hat{u}_{r}$ factors as

$$
\Sigma B_{r-1} \stackrel{\Sigma i_{r-1}}{\longrightarrow} \Sigma B_{r-1}, \Sigma B_{r-2} \stackrel{\left(u_{r-1}\right)_{*}}{\longrightarrow} B K_{p-r+2}
$$

or equivalently, $\left(\hat{u}_{r}\right)_{\#}$ factors as

$$
B_{r-1} \stackrel{i r-1}{\longrightarrow} B_{r-1}, B_{r-2} \stackrel{u_{r-1}}{\longrightarrow} K_{p-r+2} .
$$

This is proved in 7.1 using the following preparatory steps:

2.1. Fix $\lambda$-a primitive root of unity $\bmod p$. The $\lambda$-th power map on $K$ is an $\infty$-loop map hence induces self maps $T_{B_{r}}, T_{B_{r}, B_{r-1}}, \Sigma T_{B_{r-1}}=T_{\Sigma D_{r-3}}$ on $B_{r},\left(B_{r}, B_{r-1}\right), \Sigma B_{r-1}$ respectively and $i_{s, l}, j_{r}, \delta_{r}$ commute with the T's up to homotopy. Similarly the stable multiplication by $\lambda$ induces self maps $T_{E_{i}}, T_{K_{i}}, T_{\Omega K_{i}}=\Omega T_{K_{i}}$ (which are $\infty$-loops maps) on $E_{i}, K_{i}, \Omega K_{i}$ in (D1). One can easily see that in (D2) $\rangle_{p}, \tilde{u}_{p}$ commute with these self maps $T$. Choosing $W: k_{0} \circ \tilde{u}_{p} \sim u_{p} \circ j_{p}, \ell: * \sim p^{p-1} \circ k_{a}$ appropriately (for $\ell$ any stable nuil homotopy will do) one may obtain $\hat{u}_{p}$ that commutes with the T's: $\hat{u}_{p} \circ T_{\Sigma B_{p-1}} \sim T_{B K_{2}} \circ \hat{u}_{p}$. (see 5.1, 5.2, and 5.3).

Now, using self maps theory (section 5) one can make sure that in all inductive steps $(D 2)_{r} \Longrightarrow(D 2)_{r-1}$ all maps obtained commute with the self maps $T$. This translates to the algebraic fact that the classes $\left[u_{r}\right] \in H^{*}\left(B_{r}, B_{r-1}\right) \approx$ r times

$\approx \overbrace{\Sigma H^{*}(K) \otimes \cdots \otimes \Sigma H^{*}(K)}$ have algebraic weight $1 \bmod p-1$. (i.e: $\left[u_{r}\right]=$ $=\Sigma a_{i} \cdot v_{i}, v_{i}=\Sigma z_{1} \otimes \Sigma z_{2} \otimes \cdots \otimes \Sigma z_{F}, z_{i} \in H^{*}(K)$ is a monomial of primitive $z_{i}=x_{1}^{(i)} \ldots x_{\ell_{1}}^{(i)}, x_{j}^{(i)} \in P H^{*}(K)$ and $\left.\sum_{i=1}^{r} \ell_{i} \equiv 1(\bmod p-1)\right)$.

2.2. To prove 7.1 one has to show that $\hat{u}_{r} \circ \sum i_{r-2} \sim *$. The first step in the proof uses 4.3 to show that $W$ could be chosen (without violating the restriction required to obtain the properties discussed in 2.1) so that $\hat{u}_{r} \circ \sum i_{s, r-1} \sim *$.

2.3. To show that $\hat{u}_{r} \circ \sum i_{s, r-1} \sim *$ implies $\hat{u}_{r} \circ \sum i_{s+1, r-1} \sim *$ for $s<r-2$ one observes: $\hat{u}_{r} \circ \Sigma i_{s, r-1} \sim *$ is equivalent to the existence of $\hat{u}_{a}^{\prime}: \Sigma B_{s+1}, \Sigma B_{s} \longrightarrow$ $B K_{p-r+2}, \hat{u}_{a}^{\prime} \circ \sum_{j_{a+1}} \sim \hat{u}_{r} \circ i_{s+1, r+1},\left[\hat{u}_{s}^{\prime}\right]$ has an algebraic weight $1 \bmod p-1$ and $d_{s+1}\left[\hat{u}_{j}^{\prime}\right]=0$ in the cobar construction. Following computations in the cobar construction (6.2), $\left[\hat{u}_{\theta}^{\prime}\right]=d_{\theta}\left(\hat{\hat{u}}_{\theta}^{\prime}\right)$ and $\hat{u}_{r} \circ \sum i_{s+1, r-1} \sim *$.

2.4. Now one deduces the existence of (D2), for $p \geq r \geq 2$, and $\left(\hat{u}_{2}\right)_{\#}: B_{1} \longrightarrow$ $K_{p}$ represents $\phi_{p-1}\left(p^{n} i_{2 n+2}\right)$. As $\tilde{H}^{*}\left(B_{1}\right) \approx H^{*}\left(B_{1}, B_{0}\right)\left(B_{0}=*\right)$ one can write $\left(\hat{u}_{2}\right)_{\#}=u_{1}$ obtaining classes $u_{r} \in \underline{B}_{r}=H^{*}\left(B_{r}, B_{r-1}\right), 1 \leq t \leq$ $\leq p$ subject to the conditions: $u_{r}$ have $(\bmod p-1)$ algebraic weight $1, u_{p}=$ $=\overbrace{\imath \otimes \imath \otimes \cdots \otimes \imath}^{p \text { times }}, \quad p^{a_{p-++1}} u_{r}=d_{r-1} u_{r-1} . \quad$ By $6.3 \Sigma^{-1} u_{1} \in H^{*}(K)$ has the form $\varepsilon_{p} \cdot p^{1} \imath \cdot p^{2} \imath \cdot \ldots \cdot p^{p-1} \imath\left(\bmod \operatorname{Im} p^{1}\right)$ and the first part of the main theorem follows.

To show that $0 \notin \phi_{p-1}\left(P^{n} k_{z_{n+1}+1}\right)(n \not \equiv-1(p))$ suffices to show that for sorne space $X$ and some class $x \in H^{2 n+1}(X), 0 \notin \phi_{p-1}\left(p^{n} x\right) .\left(P^{n} x_{2 n+1}\right.$ is obviously in the domain of $\phi_{p-1}$ being the image of $\left.p n_{2 n+1}\right)$. This is done in Chapter 8 where some examples and applications are discussed. 


\section{The existence of $\phi_{r}$}

We use the notation of the stable version of (D1).

3.1. Proposition. If $r(r<q)$ satisfies the inequality $[r / 2]<\sum_{t=0}^{q-r-1}$ $\sum_{i=0}^{t} p,(q=2 p-2)$ then $\phi_{r}$ exists. In particular $\phi_{p-1}$ exists.

Proof: $\phi_{0}=p^{1}$ and $\phi_{1}$ obviously exist so one may assume $p>2, r \geq 1$. Now suppose $\phi_{n-1}$ exists, i.e. there exists a map $k_{n}: E_{n-1} \longrightarrow K_{n}=\Sigma^{t_{n}} K_{0}$.

The obstruction for the existence of $\phi_{n}$ is the composition $E_{n-1} \stackrel{k_{n}}{\longrightarrow} K_{n} \stackrel{p^{*} n}{\longrightarrow}$ $\Sigma K_{n+1}\left(a_{n}=1\right.$ if $n$ is even, $a_{n}=p-1$ if $n$ is odd).

Now $\Omega K_{n-1}=\Sigma^{-1} K_{n-1} \stackrel{j_{n-1}}{\longrightarrow} E_{n-1} \stackrel{k_{n-1}}{\longrightarrow} K_{n}$ is given by $p^{p-a_{n}}$, hence $* \sim p a_{n} \circ k_{n-1} \circ j_{n-1}$ and $p a_{n} \circ k_{n-1}$ factors as $E_{n-1} \stackrel{h_{n-1}}{\longrightarrow} E_{n-2} \stackrel{\omega}{\longrightarrow} \Sigma K_{n+1}=$ $=\Sigma^{t_{n+1}+1} K_{0}$.

If one can show that $H^{t_{n+1+1}}\left(E_{n-2}\right)=0,3.1$ will follow. Using the exact sequences $H^{*}\left(E_{j-1}\right) \longrightarrow H^{*}\left(E_{j}\right) \longrightarrow H^{*}\left(\Sigma^{-1} K_{j}\right)$ and $H^{*}\left(E_{0}\right)=H^{*}\left(K_{0}\right)$ suffices to prove that $H^{t_{n+1}+1}\left(\Omega K_{j}\right)=H^{t_{n+1}+1}\left(\Sigma^{t_{j}} K_{0}\right)=H^{t_{n+1}-t_{j}+2}\left(K_{0}\right)=$ $=0$ for $j \geq 0\left(t_{0}=0\right)$ provided that $[n / 2]_{p}<\sum_{t=0}^{q-n-1} \sum_{i=0}^{t} p^{i}=\lambda_{q-n+1}$. Thus, putting $t_{s}=\nu_{s} q+q-s+1, \quad t_{n+1}-t_{j}+2=m q+k$ where $m \leq$ $\nu_{n+1}, k \geq q-n+1, \nu_{n+1}= \begin{cases}\frac{n}{2} p & \text { if } n \text { is even } \\ {\left[\frac{n}{2}\right] p-1} & \text { if } n \text { is odd. }\end{cases}$

3.1. Will follow from the following:

3.2. Lemma. For $2 \leq k \leq q-1$ and $m<\sum_{t=0}^{k-2} \sum_{i=0}^{t} p^{i}=\lambda_{k}$, one has $H^{m q+k}\left(K_{0}\right)=0$. In particular the inequality holds if $m \leq \nu_{n+1} \leq\left[\frac{n}{2}\right] p<$ $<\lambda_{Q-n+1} \leq \lambda_{k}$.

Proof: Every element in $H^{m q+k}\left(K_{0}\right)$ has the form $\alpha$ where $\alpha$ is a sum of admissible monomials in $A(p),|\alpha|=m q+k$ (see $[10, p .77]$ ). An admissible monomial of $\operatorname{dim} \equiv k \bmod q$ has to contain at least $k$ Bocksteins. The lowest dimensional admissible monomial with $k$ Bocksteins is $\beta p^{k-z}+\cdots+1$ $\beta p^{p^{k-3}+\cdots+1} \ldots \beta p^{p+1} \beta P^{ \pm} \beta$ and its dimension is $\lambda_{k} q+k$.

\section{Some computations in the Steenrod algebra}

Let $\omega \in \underline{A}(p)$ be a linear combination of admissible monomials of excess $\leq 2 n+1$. (See [10]). If $P^{1} \omega$ is a sum of admissible monomials of excess $>2 n+1$ then (modulo ker $\left.P^{1}\right) \omega$ is a linear combination of elements of one of the two forms:

(i) $\omega=p^{m} \omega_{0}, m \not \equiv-I(p),\left[\left(\left|\omega_{0}\right|+1\right) / 2\right\}=m-n$.

(ii) $\omega=\left((m+1) \beta p^{m}-p^{m} \beta\right) \omega_{0}, m \neq \equiv 0,-1(p),\left[\left|\omega_{0}\right| / 2\right]=m-n$ where $\omega_{0}$ is an admissible monomial.

One has the following: 
4.1. Proposition. Let $K=K(\mathbf{Z} / p \mathbf{Z}, 2 n+1)$. Given $0 \neq u=\omega i_{2 n+1} \in$ $\in P H^{t}(k / \mathbf{Z} / p \mathbf{Z})(\omega \in \underline{A}(p))$ where

$$
t=o r \begin{cases}2 n p+1+k p q-2 k+1 & 2 \leq 2 k \leq p-1 \\
2 n p+1+k p q+s q-2 k & \left\{\begin{array}{c}
1 \leq s \leq p-1 \\
2 \leq 2 k \leq p-3
\end{array}\right.\end{cases}
$$

(the latter is void for $p=3$ ).

If $P^{1} u=0$ then $P^{1} \omega=0$ in $\underline{A}(p)$.

Proof: We may assume that excess $\omega \leq 2 n+1$ and $P^{1} u=0$ implies excess $\left(P^{1} \omega\right)>2 n+1$ hence $\omega$ has one of the two forms listed above:

(i) $u=P^{m} \omega_{0} \imath_{2 n+1},\left|\omega_{0} z_{2 n+1}\right|=2 m$ or $2 m+1$.

(ii) $u=\left((m+1) \beta^{p^{m}}-p^{m} \beta\right) \omega_{0} z_{2 n+1}, \quad\left|\omega_{0} z_{2 n+1}\right|=2 m+1$ or $2 m+2$ hence

$$
\begin{aligned}
& |u|=2 m p \quad \text { or } \quad 2 m p+1 \text { (case i). } \\
& |u|=2 m p+2 \text { or } 2 m p+3 \text { (case ii). }
\end{aligned}
$$

The hypothesis on $t$ excludes $|u|=2 m p+2, \quad|u|=2 m p$ can occur only if $t=2 n p+p q \quad(k=1), \quad\left|\omega_{0} 2_{2 n+1}\right|=2 n+q \quad$ and $\quad\left|\omega_{0}\right|=q-1$. But there are no elements in $\underline{A}(p)$ of dimension $q-1 .|u|=2 m p+1$ can occur only if $s+k=p>3$ and then $\left|\omega_{a}\right|=k q+q-2 k$ and again, there are no elements in this dimension in $\underline{A}(p)$. Similarly, $|u|=2 m p+3$ occurs only if $s+k=p-1>2$. Then $\left|\omega_{0}\right|=k q+q-2 k-1$ and there are no elements in this dimension in $\underline{A}(p)$.

4.2. Proposition. If $1 \leq a \leq p-1$ then the following sequence is exact

$$
\ldots \rightarrow \underline{A}(P) \stackrel{p^{*} \times}{\longrightarrow} \underline{A}(p) \stackrel{p^{p-s} \times}{\longrightarrow} \underline{A}(p) \stackrel{p^{*} \times}{\longrightarrow} \underline{A}(p) \rightarrow \ldots
$$

$\left(P^{a} \times\right.$ means the endomorphism of left multiplication by $\left.P^{a}\right)$.

Proof: We assume $p$-odd. The Milnor representation of $\underline{A}(p)$, [6], yields an isomorphism $\underline{A}(p) \approx P \otimes Q$. This is an isomorphism of left $P$ modules where $P$ is the subalgebra of $\underline{A}(p)$ generated by $p^{p^{i}}$. Thus, suffices to prove the exactness of the sequence where $P$ replacing $A(p)$. For $P$ one can use again the basis of admissible monomiais in the $P^{i}$ 's. Now, if $a<p$ and $\omega \in P$ is an admissible monomial then $P^{a} \omega$ is (a multiple of) an admissible monomial. If $\omega_{1} \neq \omega_{2}$ are admissible monomials then $p^{a} \omega_{1} \neq P^{a} \omega_{2}$ (unless both are zero). It follows that if $u=\Sigma_{i} k_{i} w_{i} \in \underline{A}(p), \quad k_{i} \in \mathbf{Z} / p \mathbf{Z}, \quad \omega_{i}$ admissible monomials $p^{a} u=0$ if and only if $p^{a} \omega_{i}=0$ for all $\mathrm{i}$ and suffices to prove taht $p^{a} \omega=0$ if and only if $\omega=p^{p-a} \tilde{\omega}$ for an admissible monomial $\omega$. Now $\omega=p^{m} \omega_{0}=\hat{k} p^{b} p^{m p} \omega_{0}$ for some admissible monomial $\omega_{0}, \quad 0 \leq \hat{m}, \quad 0 \leq b<p . \quad p^{a} \omega=0$ if and only if $a+b \geq p$ and then $\omega=p^{p-a}\left(\hat{k} p^{a+b-p} p^{m} p\left(\omega_{0}\right)\right.$. 
4.3. Corollary. Let $K=K(\mathbf{Z} / p \mathbf{Z}, 2 n+1)$ and let $\omega t_{2 n+1}=u \in$ $\in P H^{2 n p+1+t_{r}}(K), \omega \in \underline{A}(p)\left(t_{r}\right.$ as in 1.0-(2)). If $P^{a_{r}} u=0$ then $u=p^{a_{r+2}} u_{0}$ $\left(a_{r}\right.$ as in 1.0-(4)).

Proof: If $r$ is even $t_{r}=t_{2 k}=k p q-2 k+1,|u|=t$ as in 4.1 and $0=$ $p^{a_{r}} u=p^{1} u$ implies $p^{1} \omega=0 . \omega=p^{p-1} \omega_{0}$ and $u=p^{p-1} \omega_{0} z_{2 n+1}=p^{a+1} u_{0}$. If $r$ is odd $t_{r}=t_{2 k+1}=k p q+q-2 k$. If $0=p^{a}, u=p^{p-1} u$ then for some $s, 1 \leq s \leq p-2, p^{s-1} u \neq 0$ and $p^{1}\left(p^{s-1} u\right)=0$. The proof $u=p^{1} u_{0}$ is by induction on $s$. $\left|p^{s^{-1} u}\right|=t$ as in 4.1 and $p^{1}\left(P^{-1} u\right)=0$ implies $p^{2}\left(p^{s-1} \omega\right)=0$ (where $\left.u=\omega_{2 n+1}, \omega \in \underline{A}(p)\right)$. By $4.2 p^{s-1} \omega=p^{p-1} \omega_{0}$. If $s=1, \quad \omega=-p^{1}\left(p^{p-2} \omega_{0}\right)$ and $u=p^{1}\left(p^{p-2} \omega_{0}\right) 2_{2 n+1}$. Otherwise one has for some $\varepsilon \neq 0, \quad p^{s-1}\left(\omega-\varepsilon^{p-s} \omega_{0}\right) \imath_{2 n+1}=0$, by induction $u-\varepsilon^{p p-s} \omega_{0} t_{2 n+1}=$ $=P^{1} u_{0}$ and $u=P^{2}\left(u_{0}+\varepsilon_{1} p^{p-s^{-1}} \omega_{0} z_{2 n+1}\right)$.

\section{Review of homotopy theory of self maps}

In this section we extract some notations and statements from [12], needed in our computations. Assume all spaces and maps are pointed. Consider pairs $X, T_{X}$ of spaces $X$ with a self map $T_{X}: X \longrightarrow X$ and "morphisms" $f, V_{f}: X, T_{X} \longrightarrow Y, T_{Y}$ where $f: X \longrightarrow Y$ and $V_{f}: X \longrightarrow P Y=\operatorname{map}(I, Y)$ is a homotopy $V_{f}: f \circ T_{X} \sim T_{Y} \circ f$. Our main object here is to show that some standard homotopy theoretic constructions extend to our "category".

Now, given pairs $E, T_{E}, B, T_{B}, B_{0}, T_{B_{0}}$, morphirns $f_{0}, V_{f_{0}}: E, T_{E} \longrightarrow$ $B, T_{B}, f_{1}, V_{f_{1}}: B, T_{B} \longrightarrow B_{0}, T_{B_{0}}$ and a homotopy $\ell: * \sim f_{1} \circ f_{0} \quad(\ell: E \longrightarrow$ $\left.\underline{L} B_{0}=\operatorname{map}_{*}\left(I, 0 ; B_{0}, *\right)\right)$. We denote by $\alpha(\ell)=\alpha\left(\ell, V_{f_{0}}, V_{S_{1}}\right)$ the class of $\ell \circ T_{E}+P f_{1} \circ V_{f_{0}}+V_{f_{2}} \circ f_{0}-\underline{L} B_{0} \circ \ell$ in $\pi_{1}\left(\operatorname{map} *\left(E, B_{0}\right), *\right)$.

For convenience assume that $B_{0}=K(G, m)$ where $G$ is an elementary abelian p-group. Then one has:

5.1. Proposition ((3.9) of [12]). One can choose $\ell$ so that after replacing $T_{E}, T_{E}, T_{B_{0}}$ by their $p^{r}$-th iteration $\alpha(\ell)=0$. If $\ell$ is altered by $\omega: E \longrightarrow \Omega B_{0}$ and $\omega \circ T_{E} \sim \Omega T_{B_{0}} \circ \omega$ then $\alpha(\omega+\ell)=\alpha(\ell)$.

Similarly, suppose in addition that one is given pairs $L, T_{L}, K, T_{K}$ and maps $\sigma, V_{\sigma}: L, T_{L} \longrightarrow E, T_{E}, \quad \tau, V_{7}: K, T_{K} \longrightarrow B, T_{B}, \quad h, V_{h}: L, T_{L} \longrightarrow K, T_{K}$ and a homotopy $W: f_{0} \circ \sigma \sim \tau \circ h$, to obtain a diagram:

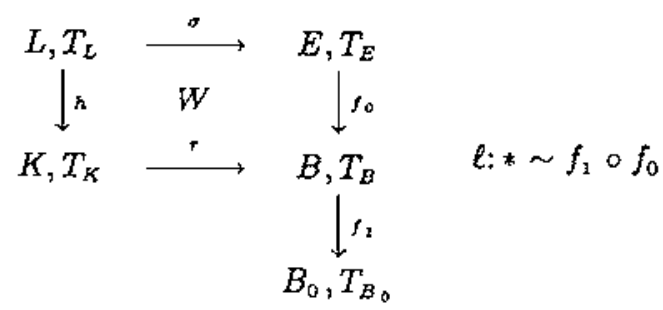


One can define an element $\alpha(W)=\alpha\left(W, V_{g}, V_{s_{0}}, V_{h}, V_{\tau}\right)$ in $\pi_{1}(\operatorname{map} *(L, B)$, $\left.f_{0} \circ \sigma \circ T_{L}\right)$ as the class of $P f_{0} \circ V_{\sigma}+V_{f_{0}} \circ \sigma+P T_{B} \circ W-V_{\tau} \circ h-P r \circ V_{h}-W \circ T_{L}$. If $B$ is an $H$-space one can translate $\alpha(W)$ to $\pi_{1}\left(\operatorname{map}_{*}(L, B), *\right)$ and then it coincides with $\alpha$ of the first type (replacing the square by maps $L \longrightarrow E \times K \longrightarrow B$ ). In this case 5.1 yields:

5.2. Proposition. $W$ can be chosen so that after replacing $T_{L}, T_{K}, T_{E}, T_{B}$ by their $p$-th iteration $\alpha(W)=0$. If $W$ is altered by $w: L \longrightarrow \Omega B$ and $w \circ T_{L} \sim$ $\sim T_{B} \circ w$ then $\alpha(w * W)=\alpha(W)$ (where ${ }^{*}$ denote the pointwise multiplication of paths in the function space).

The classes $\alpha(\ell), \alpha(W)$ represent obstructions to the following problems:

Given $f_{1}, V_{S_{1}}: B, T_{B} \longrightarrow B_{0}, T_{B_{0}}$ one naturally obtains a self map $T_{F_{f_{2}}}$ of the homotopy fiber $F_{f_{1}}$ of $f_{1}$ so that the "inclusion of the fiber map" $j_{f_{1}}: F_{f_{2}} \longrightarrow B$ strictly commutes with the self maps: $j_{f_{1}} \circ T_{F_{f_{2}}}=T_{B} \circ j_{f_{2}}$. If $\ell: * \sim f_{1} \circ$ of $f_{0} f_{0}, V_{f_{0}}: E, T_{E} \rightarrow B, T_{B}$, then $\ell$ induces a lifting $f_{\ell}: E \longrightarrow V_{f_{2}}$ of $f_{0}: j_{f_{1}} \circ$ $\circ f_{\ell}=f_{0} \cdot \alpha(\ell)=\alpha\left(\ell, V_{f_{0}}, V_{f_{1}}\right)$ is the obstruction to lift $V_{f_{0}}$ to a homotopy $V_{f_{\ell}}: f_{\ell} \circ T_{E} \sim T_{V_{f_{3}}} \circ f_{\ell}$.

Similarly, $f_{0}, V_{f_{0}}$ induces a self map $T_{C_{f_{0}}}$ on the mapping cone $C_{f_{0}}$ of $f_{0}$ so that $i_{f_{0}}: B \longrightarrow C_{f_{0}}$ strictly commutes with the self maps: $i_{f_{0}} \circ T_{B}=T_{C_{s_{0}}} \circ$ $\circ i_{f_{0}} . \ell: * \sim f_{1} \circ f_{0}$ induces a map $\hat{f}_{\ell}: C_{f_{0}} \rightarrow B_{0}$ extending $f_{1}: \hat{f}_{\ell} \circ i_{f_{0}}=$ $=f_{1} \cdot \alpha(\ell)=\alpha\left(\ell, V_{f_{0}}, V_{f_{1}}\right)$ is also the obstruction to extend $V_{f_{1}}$ to a homotopy $V_{i_{t}}: \hat{f}_{\ell} \circ T_{C}, \sim T_{B_{0}} \circ \hat{f}_{\ell}$. Analogously, $T_{K}, T_{E}, T_{B}, V_{f_{0}}, V_{r}$ in D3 induce a self map $T_{U\left(f_{0}, \tau\right)}$ on the the homotopy pull back $U\left(f_{0}, \tau\right)$ of $f_{0}$ and $\tau . W$ induces a lifting $h_{W}: L \longrightarrow U\left(f_{0}, \tau\right)$ of

$$
(\sigma \times h) \circ \Delta: L \longrightarrow E \times K .
$$

$\alpha(W)=\alpha\left(W, V_{a}, V_{f_{0}}, V_{h}, V_{\tau}\right)$ is the obstruction to lift $\left(V_{o} \times V_{h}\right) \circ \Delta$ to a homotopy $V_{h_{W}}: h_{W} \circ T_{L} \sim T_{U\left(f_{0}, \pi\right)} \circ h_{W}$. Now, if in D3 $\sigma, \tau, W, \ell$ are given, they induce a map $\rho: C_{h} \longrightarrow B_{0}$.

5.3. Proposition. Suppose in $(D s) B, B_{0}$ are $K(G, n)$ and $K\left(G_{0}, m\right)$ respectively where $G, G_{0}$ are elementary abelian p-groups. If $W: f_{0} \circ \sigma \sim \tau \circ$ oh, $\quad \ell: * \sim f_{1} \circ f_{0}$ are chosen so that $\alpha(W)=0, \alpha(\ell)=0$ then the natural map $\rho$ admits a homotopy $V_{p}: \rho \circ T_{C_{h}} \sim T_{B_{0}} \circ \rho$.

Proof: Writing down the formulas for the various maps involved one gets $(x, t \in L \times I, y \in K)$ :

$$
\begin{aligned}
& \rho(x, t)=\left\{\begin{array}{l}
\ell \sigma(x)[2 t] \\
P f_{1} \circ W(x)[2 t-1]
\end{array} \quad T_{C_{h}}(x, t)=\left\{\begin{array}{l}
T_{L}(x), 2 t \\
V_{h}(x)\{2 t-1]
\end{array}\right.\right. \\
& \rho(y)=f_{1} \circ \tau(y) \quad T_{C_{A}}(y)=T_{K}(y)
\end{aligned}
$$




$$
\left.\begin{array}{c}
\rho \circ T_{C_{h}}(x, t)=\left\{\begin{array}{l}
\ell \sigma T_{L}(x)[4 t] \\
P f_{1} \circ W \circ T_{L}(x)[4 t-1] \\
P\left(f_{1} \circ \tau\right) \circ V_{h}(x)[2 t-1]
\end{array}\right. \\
\rho T_{C_{h}}(y)=f_{1} \circ \tau \circ T_{k}(y)
\end{array}\right\}
$$

The obstruction to extend the homotopy

$P f_{1} \circ V_{\tau}+V_{f_{1}} \circ \tau: f_{1} \circ \tau \circ T_{k} \sim T_{B_{0}} \circ f_{1} \circ \tau$ to a homotopy $\rho \circ T_{C_{h}} \sim T_{B_{0}} \circ \rho$ is the class in $\pi_{1}\left(\operatorname{map}_{*}\left(L, B_{0}\right), *\right)$ of the loop

$$
\begin{aligned}
\gamma=\ell \circ \sigma \circ T_{L} & +P f_{I} \circ W \circ T_{L}+P f_{I} \circ P \tau \circ V_{h}+ \\
& +P f_{1} \circ V_{\tau} \circ h+V_{f_{1}} \circ \tau \circ h-P T_{B_{0}} \circ P f_{1} \circ W-L T_{B_{0}} \circ \ell \circ \sigma
\end{aligned}
$$

which can be illustrated as follows as a loop in $\operatorname{map}_{*}\left(L, B_{0}\right)$ :

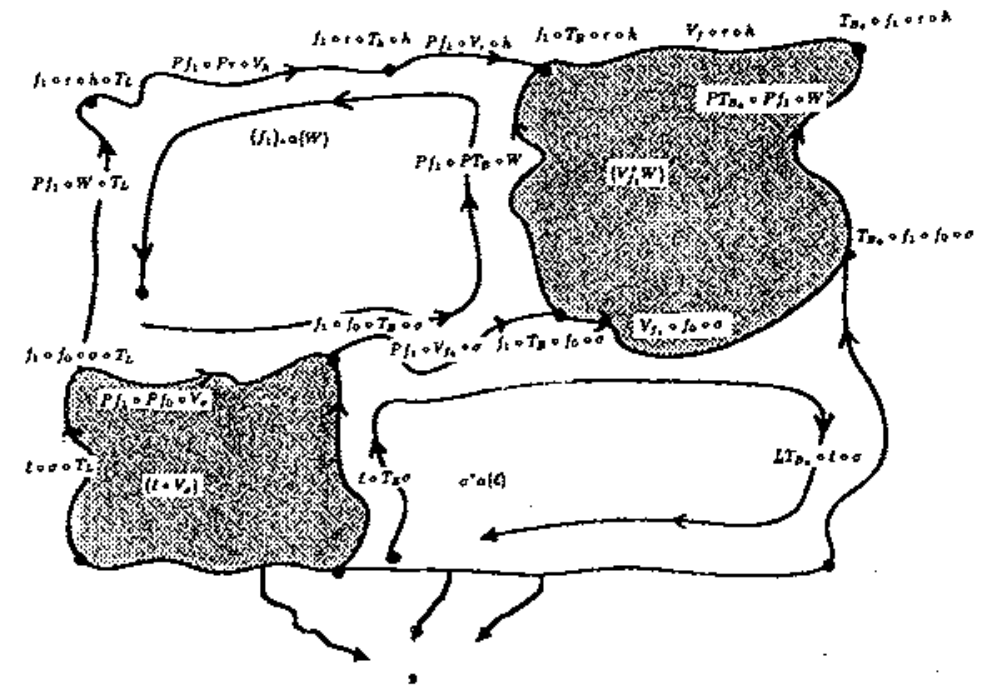

Thus $[\gamma]=-\left(\ell \circ \sigma \circ T_{L}\right)_{\# \pi_{1}}\left(f_{1} *\right) \alpha(W)+\pi_{1}\left(\sigma^{*}\right) \alpha(\sigma)$ where

$$
\begin{aligned}
& f_{1_{*}}: \operatorname{map}_{*}(L, B) \longrightarrow \operatorname{map}_{*}\left(L, B_{0}\right) \\
& \sigma^{*}: \operatorname{map}\left(E, B_{0}\right) \longrightarrow \operatorname{map}_{*}\left(L, B_{0}\right)
\end{aligned}
$$

are the left and right compositions with $f_{1}$ and $\sigma$ respectively.

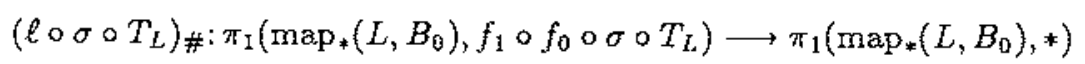

is the isomorphism induced by the path $\ell \circ \sigma \circ T_{L}$. Hence, $\alpha(W)=0, \quad \alpha(\ell)=0$ implies $[\gamma]=0$. 


\section{B. Derivations in the co-bar construction}

Let $A$ be a graded connected primitively generated Hopf algebra of finite type over $\mathbf{Z} / p \mathbf{Z}$ p-odd. Then the elements of $A$ are sums of monomials in the primitives. One has a mod $p-1$ algebraic weight function $w$ defined on homogeneous polynomials: $w(a) \in \mathbf{Z} /(p-1) \mathbf{Z}$, satisfying: $w(1)=0, W(P A)=$ $=1, w\left(\operatorname{Im}(\overbrace{P A \otimes \cdots \otimes P A}^{x_{P A}{ }^{*}} \stackrel{\Delta^{*}}{\longrightarrow} A)\right)=k(\Delta-$ the multiplication in $A)$. Thus, together with the grading this gives a bigrading of $A, A_{s, t}, s \in \mathrm{Z} /(p-$ $-1) \mathbf{Z}, t \in Z^{+}$.

Let $\underline{B}=\bigoplus_{r=0}^{\infty} \underline{B}$, be the reduced cobar construction on $A: \underline{B}_{r}=\overbrace{\bar{A} \otimes \cdots \otimes \bar{A}}^{\text {rtimes }}$ with a bigrading $\underline{B}_{r, t}=\underset{\substack{\tau_{m_{i}=t}=t \\ m_{i}>0}}{ } A_{m_{1}} \otimes \cdots \otimes A_{m}$. Then $w$ extends to $\underline{B}_{r}$ by $\omega\left(a_{1} \otimes \cdots \otimes a_{r}\right)=\sum \omega\left(a_{i}\right)$ for monomials $a_{i}$ in $A$. Let $d_{r}: \underline{B}_{r} \longrightarrow \underline{B}_{r+1}$ be the derivation $d_{r}\left(a_{1} \otimes \cdots \otimes a_{r}\right)=\sum_{i=1}^{r}(-1)^{i+1} a_{1} \otimes \cdots \otimes \bar{\mu}_{i} \otimes \cdots \otimes a_{r}$ $(\bar{\mu}$-the reduced coproduct in $A)$. Then $d_{r}$ respects the weight function thus extending it to the homology of $d_{r}$, namely to $\mathrm{Ext}_{A}^{* *}(\mathbf{Z} / p \mathbf{Z}, \mathbf{Z} / p \mathbf{Z})$. The latter is a free algebra generated by $\mathrm{Ext}_{A}^{*}(\mathrm{Z} / p Z, Z / p Z)$ and by some elements in $\bigoplus_{k} \operatorname{Ext}_{A}^{2,2 k p}(\mathbf{Z} / p \mathbf{Z}, \mathbf{Z} / p \mathbf{Z})$. All generators in $\operatorname{Ext}_{A}^{* *}(\mathbf{Z} / p \mathbf{Z}, \mathbf{Z} / p \mathbf{Z})$ are of weight one. $\left(\operatorname{Ext}_{A}^{1, m}(\mathbf{Z} / p \mathbf{Z}, \mathbf{Z} / p \mathbf{Z}) \approx P A_{m}\right.$ and the generators in $\operatorname{Ext}_{A}^{2,2 k p}(\mathbf{Z} / p \mathbf{Z}, \mathbf{Z} / p \mathbf{Z})$ are represented by $\left.\sum_{a=1}^{p-1} \frac{1}{p}\left(\begin{array}{l}p \\ a\end{array}\right) v^{a} \otimes v^{p-a}, v \in P A_{2 k}\right)$. Thus we have:

6.1. Lemma. $\operatorname{Ext}_{A}^{s, r}(\mathrm{Z} / p \mathrm{Z}, \mathrm{Z} / \mathrm{pZ})$ contains no elements of weight 1 for $2<s<p$ and for $s=2, \quad T \neq \equiv 0(\bmod 2 p)$.

6.2. Corollary. If $u \in$ ker $d_{r} \subseteq \underline{B}_{r}, w(u)=1, \quad 2<r<p$ or if $r=2$ and $|u| \not \equiv 2(\bmod 2 p)$ then $u \in \operatorname{Im} d_{r-1} \bigcap \mid$ the total degree $s+r$ in Ext $\left.{ }^{s, r}\right)$.

If $A$ above is a non stable module over $\mathbf{Z} / p \mathbf{Z}\left[P^{1}\right] /\left(P^{1}\right)^{p} \subset \underline{A}(p)$ (i.e., $p^{1} u=$ $=u^{p}$ for $u \in A_{2}, \quad P^{1} A_{1}=0$ and $P^{1}$ acts as a derivation on $a \cdot b$ and $a \otimes$ $\otimes b$. $\left.\quad P^{1}(P A) \subset P A\right)$. Then $P^{i}=\frac{t_{i}}{i !}\left(P^{2}\right)^{i} \quad(i<p)$ respects the $\bmod p-1$ algebraic weight and $P^{i}$ commuted with $d_{r}$ in $\overbrace{\bar{A} \otimes \cdots \otimes \bar{A}}^{r \text { times }} \approx \underline{B}_{r}$.

6.3. Proposition. Let $A$ be a primitively generated graded connected Hopf algebra and a module over $\mathbf{Z} / p \mathbf{Z}\left[P^{1}\right] /\left(P^{1}\right)^{p}$. Suppose $P A_{m}=0$ for $m<2 n+1$ and let $x \in P A_{2 n+1}$. Then there exist elements $u, \in \overbrace{\bar{A} \otimes \cdots \otimes \bar{A}}^{\text {. times }}$ of $\bmod p-1$ algebraic weight $1, \quad 1 \leq s \leq p$, so that:

(i) $u_{p}=\overbrace{x \otimes x \otimes \cdots \otimes x}^{p \text { times }}$ $p_{o+1} u_{s}=d_{s-1} u_{s-1}$ for $s>1$ 
$(u_{s-1} \in \overbrace{\overbrace{A}^{-1} \otimes \cdots \otimes \bar{A}}^{\text {times }}$ considered as an element in $\underline{B}_{s-1}) a_{s+1}$ as in 1.0 (4). For any choice of $u$, of weight 1 (modp-1) satisfying (i) and (ii) one has $u_{1}=\varepsilon_{p} x \cdot p^{2} x \cdot p^{2} x \ldots p^{p-1} x\left(\bmod \operatorname{Im} p^{1}\right) \varepsilon_{p}=(-2)^{p-\frac{1}{2}}$.

Proof: Suppose by induction $u_{p-r}$ of weight one were found for $0 \leq r \leq r_{0}$ so that, $u_{p}=x \otimes \cdots \otimes x$ and $p^{a_{r}} u_{p-r+1}=d_{p-r} u_{p-r},\left|u_{\downarrow}\right|=$ odd. Further assume:

$$
\text { (for } \quad r=2 s) \underset{p-2 s-1 \text { times }}{u_{p-2 s}}=\left[p^{p-1}\left(p^{1} x \cdot x\right)\right]^{s} \cdot x \overbrace{\otimes x \otimes \cdots \otimes x}^{p-2 s-1 \text { times }}+\sum_{i} w_{1}^{i} \otimes w_{2}^{i}
$$

where $w_{2}^{i} \in \overbrace{A \otimes \cdots \otimes A}^{A \otimes \cdots}, \quad\left|w_{2}^{i}\right|>(2 n+1)(p-2 s-1)$

$$
\begin{aligned}
(\text { for } r=2 s+1) \quad u_{p-2,-1}= & \{\left.p^{p-1}\left(p^{1} x \cdot x\right)\right|^{s}\left(p^{1} x \cdot x\right) \otimes \overbrace{x \otimes \cdots \otimes x}^{p-2 s-2 \text { times }}+ \\
& +\sum_{i} v_{1}^{i} \otimes v_{2}^{i}
\end{aligned}
$$

where $v_{2}^{i} \in \overbrace{A \otimes \cdots \otimes A}^{p-2,-2 \text { im es }}, \quad\left|v_{2}^{i}\right|>(2 n+1)(p-2 s-2)$.

$$
\text { If } r_{0}=0, \quad p^{1} u_{p}=p^{1}(x \otimes \cdots \otimes x)=\sum_{i=1}^{p} x \otimes \cdots \otimes p^{1} x \otimes \cdots \otimes x=
$$
$=d_{p-1} \sum_{i=1}^{p-1}\left((-1)^{i+1} \cdot i\right) x \otimes \cdots \otimes p^{1} x \cdot x \otimes \cdots \otimes x$ and $u_{p-1}$ exists having the desired form. If $\tau_{0}=2 s_{0}+1, \quad s_{0} \geq 0, \quad p^{1} u_{p-2 s_{0}}=d_{p-2 s_{0}-1} u_{p-2 s_{0}-1}$ imply $d_{p-2 s_{0}-1} p^{p-1} u_{p-2 s_{0}-1}=0$. As $w\left(p^{p-1} u_{p-2 s_{0}-1}\right)=1 \quad p>p-2 s_{0}-1>$ $>1,\left|p^{p-1} u_{p-2 s_{0}-1}\right|=$ odd, hence $\neq 2(\bmod 2 p)$, by $6.2 p^{p-1} u_{p-2 s_{0}-1} \in$ $\in \operatorname{Im} d_{p-2 s_{0}-2}$.

Now $p^{p-1} u_{p-2 s_{0}-1}=\left[p^{p-1}\left(p^{1} x \cdot x\right)\right]^{0+1} \otimes \overbrace{x \otimes x \cdots \otimes x}^{p-2 s-2 \text { times }}$ $+\sum_{i} \tilde{v}_{1}^{i} \otimes \tilde{v}_{2}^{i},\left|\tilde{v}_{2}^{i}\right|>(2 n+1)(p-2 s-2)$. One can easily argue that if $p^{p-1} u_{p-2 s_{0}-1}=d_{p-2 s_{0}-2} u_{p-2 s_{0}-2}$, then $u_{p-2 s_{0}-2}$ has the desired leading term (modker $\left.d_{p-2 s_{0-2}}\right)$. If $r_{0}=2 s_{0}, 0<s_{0}<\frac{p-1}{2}, p^{p-1} u_{p-2 s_{0}+1}=$ $=d_{p-2 s_{0}} u_{p-2 s_{0}}$ and $d_{p-2 s_{0}} p^{1} u_{p_{p-2 s_{0}}}=0$. As $p>p-2 s_{0}>1, \quad\left|p^{1} u_{p-2 s_{0}}\right|=$ $=\operatorname{odd} \not \neq 2(\bmod 2 p)$, again $p^{1} u_{p-2 s_{0}} \in \operatorname{Im} d_{p-2 s_{0}-1} \cdot p^{1} u_{p-200}=$ $=\left[p^{p-1}\left(p^{2} x \cdot x\right)\right]^{0} p^{1} x \otimes \overbrace{x \otimes \cdots \otimes x}^{p-2 *-1 \text { times }}+\sum_{i} \tilde{w}_{1}^{i} \otimes \tilde{w}_{2}^{i}\left|\tilde{w}_{2}^{i}\right|>(p-2 s-1)(2 n+1)$. Again one can see that $p^{1} u_{p-2 s_{0}}=d_{p-2 s_{0}-1} u_{p-2 s_{0}-1}, u_{p-2 s_{0}-1}$ has the desired leading term (mod $\left.\operatorname{Im} d_{p-2 *_{0}-1}\right)$.

Now, one can see by induction that $u_{p-\psi}$ are uniquely determined $\bmod \left(\operatorname{ker} d_{p-r}=\operatorname{Im} d_{p-r-1}\right)+\operatorname{Im} \rho^{t+1}$. Thus $u_{1}$ is determined up to image $p^{a_{2}}=\rho^{1}$ and $u_{1}=\left\{p^{p-1}\left(p^{1} x \cdot x\right)\right\}^{p-\frac{1}{2}} \cdot x=\varepsilon_{p} x p^{1} x \ldots p^{p-1} x \bmod \operatorname{Im} p^{1}$. 


\section{Proof of the main theorem, part (1)}

Fix $p$ odd and $n \not \neq-1(\bmod p)$. We follow the notations of 2.0 (i) -(iv) and recall the observation that $H^{*}\left(B_{r}, B_{r-1}\right) \approx \underline{B}_{r} A$ where $A=H^{*}(K), \underline{B}_{r} A \approx$

$\approx \overbrace{\Sigma A \otimes \cdots \otimes \Sigma A}^{\text {rimes }}\left(\Sigma A=\tilde{H}^{*}(\Sigma K)\right)$. The composition $B_{\mathrm{r}+1}, B_{r} \stackrel{\delta_{r}}{\longrightarrow} \Sigma B_{r} \stackrel{\Sigma j_{r}}{\longrightarrow}$ $\Sigma B_{r}, B_{r-1}$ induces a morphism $H^{*}\left(B_{r}, B_{r-1}\right) \longrightarrow H^{*}\left(B_{r+1}, B_{r}\right)$ corresponding to the derivation in the cobar construction

$$
\begin{gathered}
d_{r}: \overbrace{\bar{A} \otimes \bar{A} \otimes \cdots \otimes \bar{A}}^{r \text { times }} \longrightarrow \overbrace{\bar{A} \otimes \cdots \otimes \bar{A}}^{r+1}, \quad d_{r}\left(a_{1} \otimes \cdots \otimes a_{r}\right)= \\
=\sum_{j=1}^{r}(-1)^{j} a_{1} \otimes \cdots \otimes \bar{\mu}^{*} a_{j} \otimes \cdots \otimes a_{r}
\end{gathered}
$$

where $\bar{\mu}^{*}: \bar{A} \longrightarrow \bar{A} \otimes \bar{A}$ is the reduced coproduct (note that $\bar{A} \approx \Sigma A$ with a shift of dimensions).

Now, starting of with (D2), one observes that all maps (except possibly $\hat{u}_{p}$ ) commute with the self maps $T$ induced by the $\lambda$-th power maps in $K$ (and consequently in $\left.\Sigma K, B_{i},\left(B_{i}, B_{i-1}\right)\right)$ and by those in $E_{i}, K_{i}$. One can see that if $\ell: * \sim p^{p-1} \circ k_{0}, V_{k_{0}}: k_{0} \circ T_{E_{0}} \sim T_{K_{1}} \circ k_{0}, V_{p y-1}: p^{p-1} \circ T_{K_{1}} \sim T_{B K_{3}} \circ p^{p-1}$ are chosen to be the stable homotopies then $\alpha\left(\ell, V_{k_{0}}, V_{p p-2}\right)=0$.

By 5.2, 5.3 one can choose $W: k_{0} \circ \tilde{u}_{p} \sim u_{p} \circ j_{p}$ so that $\hat{u}_{p}: \Sigma B_{p-1} \longrightarrow B K_{2}$ will satisfy $\hat{u}_{p} \circ T_{\Sigma B p-1} \sim T_{B K}, \circ \hat{u}_{p}$ (where the T's may have to be replaced by their $p^{t}$-th iteration).

The same observation will hold in all the following inductive steps: If (D2), is given so that all maps (with the possible exception of $\hat{u}_{r}$ ) commute up to homotopy with the self maps $T$ then one chooses the stable homotopies $\ell: * \sim$ $\sim p^{a_{p-r+1}} \circ k_{p-r}, \quad V_{k_{p-r}}: k_{p-r} \circ T_{E_{p-r}} \sim T_{K_{p-1+1}} \circ k_{p-r}, \quad V_{p a_{p-r+1}}: p^{a_{p-p+1}}$ $\circ T_{K_{p-+1}} \sim T_{B K_{p+r+2}} \circ p^{a,-r+2}$ and a suitable $W: k_{p-r} \circ \tilde{u}_{r} \sim u_{r} \circ j_{r}$ so that $\hat{u}_{r}$ induced by $\ell$ and $W$ will satisfy $\hat{u}_{r} \circ T_{B r-2} \sim T_{B K_{s-r+}} \circ u_{r}$. Thus if one assumes inductively (induction on $p-r$ ) that (D2), exists for all $p \geq r \geq r_{0} \geq 3$ so that all maps commute up to homotopy with the self maps $T$ one has to prove the following proposition in order to continue the inductive process:

7.1. Proposition. $\hat{u}_{\mathrm{r}_{0}} \circ \sum i_{\mathrm{r}_{0}-2} \sim *$.

If 7.1 is proved then $\hat{u}_{r_{0}}$ may be factored as

$$
\Sigma B_{r_{0}-1} \stackrel{\Sigma j_{r_{0}-2}}{\longrightarrow} \Sigma B_{r_{0}-1}, \Sigma B_{r_{0}-2} \stackrel{\left(u_{r_{0}-1}\right)_{*}}{\longrightarrow} B K_{p-r_{0}+2}
$$

and $\left(u_{r_{0}-1}\right)_{*}$ commutes with the T's (5.1). The same holds for the adjoint $\operatorname{map} u_{r_{0}-1}: B_{r_{0}-1}, B_{r_{0}-2} \longrightarrow K_{p-r_{0}+2}$. 
By induction $\left[k_{p-r_{0}} \circ \tilde{u}_{r_{0}}\right]$ represents $\Phi_{p-r_{0}}\left(p^{n} l_{2 n+2}\right)$ and consequently $\Phi_{p-r_{0}+1}\left(p^{n} 2_{2 n+2}\right)$ is represented by $\left[\left(\hat{u}_{r_{0}}\right)_{\#}\right] \in\left[B_{r_{0}-1}, K_{p-r_{0}+2}\right]$, in particular $\left(\hat{u}_{r_{0}}\right)_{\#}$ factors as

$$
B_{r_{0}-1} \stackrel{\tilde{u}_{r_{0}-1}}{\longrightarrow} E_{p-r_{0}+1} \stackrel{k_{p-r_{0}+1}^{\longrightarrow}}{\longrightarrow} K_{p-r_{0}+2}: \tilde{u}_{r_{0}-1}
$$

is the lifting of $\tilde{u}_{r_{0}} \circ i_{r_{0}-1}$ induced by $\underline{L} u_{r_{0}} \circ \ell_{0}+W \circ i_{r_{0}-1}: * \sim k_{p-r_{0}} \circ \tilde{u}_{r_{0}} \circ i_{r_{0}-1}$ where $\ell_{0}$ is the standard homotopy $\ell_{0}: * \sim j_{r_{0}} \circ r_{r_{0}}$. One can easily see that $\alpha\left(\ell_{0}\right)=0$ and $W$ was chosen to have $\alpha(W)=0$, hence, by a dual of 5.3 $\tilde{u}_{r_{0}-1}$ commutes with the self maps. Applying 7.1 one obtains $(D 2)_{r_{0-1}}$ as required. This inductive process yields diagrams $(D 2)$, for $2 \leq r \leq p$. If one denotes the adjoint of $\hat{u}_{2}: \Sigma B_{1} \rightarrow B K_{p}$ by $u_{1}: B_{1} \rightarrow K_{p}$ (and as all maps are pointed $u_{1}: B_{1}, B_{0} \longrightarrow K_{p}$ where $\left.B_{0}=*\right)$ then $\left\{u_{1}\right]$ represents $\Phi_{p-1}\left(P^{n} t_{2 n+2}\right)$. In this way one obtains a sequence of maps $\left[u_{r}\right] \in\left[B_{r}, B_{r-1} ; K_{p-r+1}\right]=$ $=H^{2 n p+2+t_{p-r+1}}\left(B_{r}, B_{r-1}\right)$ satisfying:

(i) $u_{p}=\imath_{2 n+2} \otimes \cdots \otimes \imath_{2 n+2}$,

(ii) $p a_{p-r+1}\left[u_{r}\right]=\left[u_{r-1} \circ \Sigma i_{r-1} \circ \delta_{r}\right]$.

One shifts dimensions and considers $\Sigma^{-r} u_{\mathrm{r}} \in \overbrace{\bar{A} \otimes \cdots \otimes \bar{A}}^{\text {rimes }}=\underline{B}_{r}\left(\bar{A}=\bar{H}^{*}(K)\right)$ then (i), (ii) are shifted to satisfy the hypothesis of 6.3 ( $u_{r}$ of $\bmod p-1$ algebraic weight 1 is equivalent to the property that $u_{r}$ commutes with the self maps). Hence, $\Sigma^{-1} u_{1}=\varepsilon_{p} \imath_{2 n+1} \cdot p^{1} \imath_{2 n+1} \ldots p^{p-1} \imath_{2 n+1}\left(\bmod \operatorname{Im} p^{1}\right)$. As $\operatorname{Im} p^{1}$ is part of the indeterminancy of $\Phi_{p-1}$ part (1) of the main theorem follows.

Proof of 7.1: First consider the composition:

$$
\Sigma B_{1} \stackrel{\Sigma i_{1, r_{0}-1}}{\longrightarrow} \Sigma B_{r_{0}-1} \stackrel{\tilde{u}_{r_{0}}}{\longrightarrow} B K_{p-r_{0}+2} \quad\left(r_{0} \geq 3\right) .
$$

Now

$$
\tilde{H}^{*}\left(\Sigma B_{1}\right) \approx H^{*}\left(\Sigma B_{1}, \Sigma B_{0}\right) \approx \Sigma^{2} \tilde{H}(K) \approx \Sigma^{2} \bar{A}
$$

and the image of $H^{*}\left(\Sigma i_{1, r_{0}-1}\right)$ is $\Sigma^{2} P A$. Now, $\left(\hat{u}_{r_{0}}\right)_{\#}: B_{r_{0}-1} \longrightarrow K_{p-r_{0}+2}$ represents $\Phi_{p-r_{0}+1}\left(P^{n} t_{2 n+2}\right)$, hence, $* \sim P^{a}, r_{0} \circ \hat{u}_{r_{0}}$ and $P^{a-r_{0}}\left[\hat{u}_{r_{0}} \circ \Sigma i_{1, r_{0}-1}\right]=$ $=0, \quad\left[\hat{u}_{r_{0}} \circ \Sigma i_{1, r_{0}-1}\right] \in \Sigma^{2} P A$. Let $u=\Sigma^{-2}\left[\hat{u}_{r_{0}} \circ \Sigma i_{i_{1, r_{0}-1}}\right] \in P A$ then $u$ has weight $1(\bmod p-1)$ and its dimension is $2 n p+1+t_{p-r_{0}}$. By 4.3 $u=p^{a_{p-r_{0}+1}} v, v \in P A$. Now the map $H^{*}(B K)=H^{*}\left(B_{\infty}\right) \longrightarrow H^{*}\left(B_{1}\right) \stackrel{\Sigma^{-1}}{\longrightarrow}$ $H^{*}(K)$ induces a surjection $P H^{*}(B K) \rightarrow P H^{*}(K)$ and $\Sigma^{2} v$ belongs to $\operatorname{Im} H^{*}\left(\Sigma B_{r_{0}}\right) \rightarrow H^{*}\left(\Sigma B_{1}\right)$, say $\Sigma^{2} v=H^{*}\left(i_{1, r_{0}}\right) \tilde{v}$. Thus one can alter $W: k_{p-r_{0}} \circ \tilde{u}_{r_{0}} \sim u_{r_{0}} \circ j_{r_{0}}$ by $\tilde{v} \in\left[\Sigma B_{r_{0}}, K_{p-r_{0}+1}\right]$ (as $\tilde{v}$ is of weight 1 it commutes with the self maps, by $5.2 \alpha((-v) * W)=0)$ and $\tilde{u}_{r_{0}}$ is thus altered to obtain $\tilde{u}_{r_{0}} \circ \Sigma i_{1, r_{0}-1} \sim *$.

Suppose inductively that $\hat{u}_{r_{0}} \circ \Sigma i_{r_{,} r_{0}-1} \sim *$ for some $r, \quad 1 \leq r<r_{0}-2$. Then the composition $\Sigma B_{r} \rightarrow \Sigma B_{\mathrm{r}+1} \rightarrow \Sigma B_{\mathrm{r}_{0}-1} \stackrel{\overline{\mathrm{u}}_{\mathrm{o}}}{\longrightarrow} B k_{\mathrm{p}-\mathrm{r}_{\mathrm{o}}+2}$ is null homotopic. We also assume that all maps commute with the self maps $T$. 
By 5.1 (and by replacing the self maps by their $p^{t}$ iterations, if necessary) $u_{\mathrm{r}_{9}} \circ$ o $\Sigma i_{r+1, r_{0}-1}$ extends to a map $\Sigma B_{r+1}, \Sigma B_{r} \stackrel{u_{r}^{\prime}}{\longrightarrow} B K_{p \rightarrow r_{q}+2}$ which homotopically commutes with the self maps. The $\lambda$ power map induces a multiplication by $\lambda$ on the fundamental class of $B K_{p-s_{0}+2}$, the fact that $u_{r}^{\prime}$ commutes with the self maps is equivalent to the fact that $\left[u_{r}^{\prime}\right] \in H^{*}\left(\Sigma B_{\mathrm{r}+1}, \Sigma B_{r}\right)$ is a $\lambda$ characteristic vector of $H^{*}\left(T_{\Sigma B_{r+1}, \Sigma_{B}}\right)$, hence it is a class of $\bmod p-1$ algebraic weight 1. $r<\tau_{0}-2$ implies that the composition $B_{r+1}, B_{r} \stackrel{\sigma_{r+3}}{\longrightarrow} \Sigma B_{r+1} \rightarrow B_{r_{0}-1}$ is null homotopic, hence, $\hat{u}_{r}^{t} \circ \sum j_{r+1} \circ \delta_{r+1} \sim \hat{u}_{r_{0}} \circ \sum i_{r+1, r_{0}-1} \circ \delta_{r+1} \sim *$, $d_{r+1}\left[\Sigma^{-1} \hat{u}_{r}^{\prime}\right]=0$ in the cobar construction. As $p-1 \geq r+1 \geq 2$ and $\left|\Sigma^{-1} \hat{u}_{2}^{\prime}\right|=2 n p+2+t_{p-r_{0}+2} \not \equiv 2 \bmod 2 p$ by $6.2 \Sigma^{-1} \hat{u}_{r}^{\prime} \in \operatorname{Im} d_{r}, \hat{u}_{r}^{\prime} \sim$ $\sim \hat{\hat{u}}^{\prime}, \circ \Sigma^{2} j_{r} \circ \Sigma \delta_{r+1}$,

$$
\Sigma B_{r+1}, \Sigma B_{r} \stackrel{\Sigma \delta_{r+3}}{\longrightarrow} \Sigma^{2} B_{r} \stackrel{\Sigma j_{r}}{\longrightarrow} \Sigma^{2} B_{r}, \Sigma^{2} B_{r-1} \stackrel{\hat{\theta}_{r}}{\longrightarrow} B K_{p-r_{0}+2}
$$

hence, $\hat{u}_{r_{0}} \circ \sum i_{r+1, r_{0}-1} \sim \hat{\hat{u}}_{r}^{\prime} \circ \sum j_{r+1} \sim \hat{\hat{u}}_{r} \circ \Sigma^{2} j_{r} \circ \Sigma \delta_{r+1} \circ \sum j_{r+1} \sim *$. By induction $\hat{u}_{r_{0}} \circ \sum i_{r_{r} r_{0}-1} \sim *$ for all $r \leq r_{0}-2, \quad \hat{u}_{r_{0}} \circ \sum i_{r_{0}-2, r_{0}-1}=\hat{u}_{r_{0}} \circ \sum i_{r_{0}-2} \sim$ $\sim *$ and 7.1 follows.

\section{The main theorem (part (2)) and applications}

The proof of the main theorem (2) (see 8.3) is a consequence of some applications of the main theorem part (1).

8.1. Proposition. Let $\lambda$ be a primitive root of unity $\bmod p$. Let $X, T$ be a $Q-\lambda$ power space, i.e. $T: X \rightarrow X$ induces multiplication by $\lambda$ on the quotient module $Q H^{*}(X)$ of algebra generators. If for some $n, n \not \equiv-1$ $\bmod p, Q H^{i}(X)=0$ for all $i \neq 2 n+1$ mod $p-1$ and if $x \in H^{2 n+1}(X)$ is an indecomposable $\lambda$ characteristic vector of $H^{*}(T)$ then $\Phi_{p-1}\left(P^{n} x_{2 n+1}\right)=$ $=\varepsilon_{p} x \cdot p^{1} x \ldots p^{p-1} x+\operatorname{Im} p^{1}+\bigoplus_{i \neq 1} W_{i}$ where $W_{i} \subset H^{\cdot}(X)$ is the $\lambda^{i}$ eigenspace, $1 \leq i \leq p-1$. (One may assume that $H^{m}(T)$ has a diagonal form for every $m$ ).

Proof: $\Sigma T$ induces a mod $p$ splitting of $\Sigma X, \quad \Sigma X \approx_{p} \bigvee_{i=1}^{p-1} Y_{i}, H^{\cdot}\left(Y_{i}\right)=$ $=\Sigma W_{i} . \quad\left(Y_{i_{0}}\right.$ is the mapping telescope of $\left.\prod_{i \neq i_{0}^{j}}\left(\Sigma T-\lambda^{i}\right)\right) . \quad p^{n} x$ is obviously in the domain of $\Phi_{p-1}$. A representative of $\Phi_{p-1}\left(p^{n} x\right)$ could be obtained by $X \stackrel{x}{\rightarrow} K(\mathbf{Z} / p \mathbf{Z}, 2 n+1) \stackrel{\left(u_{1}\right)_{\#}}{\longrightarrow} E_{p-1} \stackrel{k_{p}}{\longrightarrow} K_{p}$, hence, by the main theorem (1) $\varepsilon_{p} x \cdot p^{1} x \ldots p^{p-1} x \in \Phi_{p-1}\left(p^{n} x\right)$. Let $: X \stackrel{j}{\rightarrow} E_{p-1} \stackrel{k_{p-1}}{\longrightarrow}$ $\stackrel{k_{p-3}}{\longrightarrow} K_{p}$ be any other representative of $\Phi_{p-1}\left(P^{n} x\right)$, hence the composition $X \stackrel{i}{\rightarrow} E_{p-1} \stackrel{h_{2} \circ \cdots \circ h_{p-1}}{\longrightarrow} K(\mathbf{Z} / p \mathbf{Z}, 2 n p+1)$ is $p^{n} x$. Denote $f=\left(u_{1}\right)_{\#} \circ x$ then 
$\hat{f}_{\#}-f_{\#}: \Sigma X \longrightarrow B E_{p-1}$ satisfies $B\left(h_{1} \circ \cdots \circ h_{p-1}\right) \circ\left(\hat{f}_{\#}-f_{\#}\right) \sim *$ and $\hat{f}_{\#}-f_{\#}$ lifts to $\Sigma X \rightarrow B \hat{E}_{p-1}$. $\left(\hat{E}_{p-1}\right.$ the homotopy fiber of $\left.h_{1} \circ \ldots h_{p-1}\right)$. Now one can easily see that $\pi_{2 n p+1+i}\left(\hat{E}_{p-1}\right)=0$ for $i \equiv 0(p-1), 0<i<t_{p-1}-1$. On the other hand $W_{1} \subset \bigoplus_{0} H^{2 n+1+(p-1) \circ}(X)$ (as $W_{1}$ is spanned by monomials in the indecomposable $\lambda$-eigenvectors of length $\equiv 1 \bmod p-1$ ). It follows that the map $\hat{j}_{p-1}: \Omega K_{p-1} \longrightarrow \hat{E}_{p-1}$ (the lifting of $\hat{j}_{p-1}$ in (D1)) induces a surjection $\left[Y_{1}, K_{p-1}\right] \longrightarrow\left[Y_{1}, B \hat{E}_{p-1}\right]$. Hence, $Y_{2} \stackrel{i_{2}}{\longrightarrow} \Sigma X \stackrel{\hat{f}_{*}-f_{*}}{\longrightarrow} B E_{p-1} \stackrel{B k_{p-1}}{\longrightarrow} B K_{p}$ factors as $Y_{1} \stackrel{\omega}{\rightarrow} K_{p-1} \stackrel{p^{2}}{\longrightarrow} B K_{p}$, and $\left[k_{p} \circ \hat{f}\right]-\left[k_{p} \circ f\right]=p^{2}\left(\Sigma^{-1} \omega\right)+\bigoplus_{i \neq 1} W_{i}$. Hence $\left[k_{p} \circ \hat{f}\right]=\varepsilon_{p} x \cdot p^{1} x \ldots p^{p-1} x+\operatorname{In} p^{1}+\bigoplus_{i \neq 1} W_{i}$.

8.2. Example. Let $X=B(2 n+1)$ be the $2 n$ connected Wilson's irreducible factor of the $2 n$ connected element of the $\Omega$-spectrum of $B P$. If $n \not \equiv-1 \bmod p$ then for the fundamental class $x \in H^{2 n+1}(X)$ one has $\varepsilon_{p} x \cdot p^{1} x \ldots p^{p-1} x \notin$ $\notin \operatorname{Im} P^{1}+\bigoplus_{i \neq 1} W_{i}$ hence, by $8.10 \notin \Phi_{p-1}\left(p^{n} x\right)$.

8.3. Proof of the main theorem (part(2)): $0 \in \Phi_{p-1} p^{n} \imath_{2 n+1}$ will contradict 8.2 .

8.4. Corollary. Let $X, T, n, x$ be as in 8.1. If $0 \in \Phi_{p-1}\left(P^{n} x_{2 n+1}\right)$ (in particular if $p^{n} x_{2 n+1}=0$ ) then $x \cdot p^{1} x \ldots p^{p-1} x \in \operatorname{Im} p^{1}$.

Proof: By 8.1, $0 \in \Phi_{p-1}\left(p^{n} x\right)=\varepsilon_{p} x^{p^{1}} x \ldots p^{p-1} x+\operatorname{Im} p^{1}+\bigoplus_{i \neq 1} W_{i}=$ $=\varepsilon_{p} x P^{1} x \ldots p^{p-1} x+P^{1} W_{1}+\bigoplus_{i \neq 1} W_{i}$. As $x P^{1} x \ldots P^{p-1} x \in W_{1}, P^{1} W_{1} \subset W_{1}$ and $W_{1} \cap \bigoplus_{i \neq 1} W_{i}=0$ one has $x P^{1} x \ldots P^{p-1} x \in P^{1} W_{1}$.

8.5. Corollary. There is no $Q-\bar{\lambda}$ power space $X$ having $H^{*}(X)=$ $=\wedge\left(x, p^{1} x, \ldots, p^{p^{-1}} x\right), \operatorname{dim} X=2 n+1, n \not \equiv-1(\bmod p)$.

Proof: $P^{n} x=0$ by hypothesis but there are no elements $w$ with $P^{1} w=$ $=x \cdot P^{1} x \ldots p^{p-1} x$ to satisfy the conclusions of 8.4

8.5.1. Remark. If $n$ is large enough $\left(n>\frac{1}{2}\left(p^{2}-p-1\right)(p-1)\right)$ then there is no space (not necessarily a power space) with $H^{*}(X)=\wedge\left(x, p^{1} x, p^{2} x, \ldots, p^{p-1} x\right)$. This follows from the fact that $H^{m}(X)=0$ for $m \not \equiv 2 n+1(\bmod p-1)$ in the relevant range (as in the proof of 8.1 ), hence, $\operatorname{Im} p^{1}$ is the total indeterminancy. In [3] a mod $p(p \geq 5) H$-space is constructed with $H^{*}(X)=\wedge\left(x, P^{ \pm} x, \ldots\right.$ $\left.\ldots, p^{p} x\right\rangle,|x|=2 p+1$.

8.6. Corollary. There is no stable map $f: X \longrightarrow \Omega^{\infty} \sum^{\infty} S^{2 p^{2}+1}$ with $p^{p} x \in$ $\in \operatorname{Im} H^{*}(f)$.

Proof: As $\Phi_{p-1}$ is stable $0 \in \Phi_{p-1}(u)$ where $0 \neq u \in H^{2 p^{2}+1}\left(\Omega^{\infty} \Sigma^{\infty} S^{2 p^{2}+1}\right)$. A map $f$ with $H^{*}(f) u=p^{p} x$ will yield (by 8.4) $x \cdot p^{1} x \ldots p^{p-1} x \in \operatorname{Im} p^{1}$ which is impossible. 


\section{References}

1. J.F. ADAMS, On the non existence of elements of Hopf invariant one, $A n n$. of Math. 72 (1960), 20-104.

2. G.E. COOKE AND L. SMITh, Descomposition of co H-spaces and applications, Math. Z. 157 (1977), I55-157.

3. J.HARPER AND A. ZABRODSKY, H-spaces and self maps, in "Algebraic Topology-Aarhus 1982 Springer LN 1051," pp. 371-373.

4. H. MILLER, Stable Splittings of Stiefel manifolds, (Mimeographed).

5. J. MiLNoR, Construction of universal bundles II, Ann. of Math. 63 (1956), 430-436.

6. J: MiLnor, The Steenrod algebra and its dual, Ann. of Math. 67 (1958), 150-171.

7. T. NiSHIDA, On a result of Sullivan and the $\bmod p$ decomposition of Lie groups, Research Inst. for Math. Sic. Kyoto \$ (1971).

8. M. ROTHENBERG AND N. E. STEENROD, The cohomology of classifying spaces of H-spaces, Bull AMS 71 (1965), 872-875.

9. J. STASHEFF, H-spaces problems, in "Neuchatel 1970 Springer LN 196," pp. 122-136.

10. N.E. Steenrod AND D. B. A. EPstein, "Cohomology operations," Ann. of Math. Studies 50, 1962.

11. A. ZABRODSKY, Some relations in the $\bmod 3$ cohomology of $\mathrm{H}$-spaces, Israel J. of Math. 33 (1979), 59-72.

12. A. ZABRODSKY, Endomorphism in the homotopy category, in "Combinatorial Methods in topology and algebraic geometry," Contemporary Mathematics, pp. 227-277.

J. Harper: Department of Mathematics

The University of Rochester, U.S.A.

A. Zabrodsky: Institute of Mathematics

Hebrew University of Jerusalem, ISRAEL.

Rebrut el 20 d'Octubre de 1986 